\title{
Global trends in myopia management attitudes and strategies in clinical practice
}

\author{
James S. Wolffsohn ${ }^{\mathrm{a}, *}$, Antonio Calossi ${ }^{\mathrm{b}}$, Pauline Cho ${ }^{\mathrm{c}}$, Kate Gifford ${ }^{\mathrm{d}}$, Lyndon Jones ${ }^{\mathrm{e}}$, \\ Ming Lif ${ }^{\mathrm{f}}$, Cesar Lipener ${ }^{\mathrm{g}}$, Nicola S. Logan ${ }^{\mathrm{a}}$, Florence Malet ${ }^{\mathrm{h}}$, Sofia Matos ${ }^{\mathrm{i}}$, \\ Jose Manuel Gonzalez Meijome ${ }^{\mathrm{i}}$, Jason J. Nichols ${ }^{\mathrm{j}}$, Janis B. Orr ${ }^{\mathrm{a}}$, \\ Jacinto Santodomingo-Rubidok ${ }^{\mathrm{k}}$, Tania Schaefer ${ }^{\mathrm{l}}$, Nilesh Thite ${ }^{\mathrm{m}}$, Eef van der Worp ${ }^{\mathrm{n}}$, \\ Madara Zvirgzdina ${ }^{a}$
}

a Ophthalmic Research Group, School of Health E Life Sciences, Aston University, Birmingham, UK

${ }^{\mathrm{b}}$ Department of Physics (Optics and Optometry), University of Florence, Italy

' School of Optometry, The Hong Kong Polytechnic University, Hong Kong Special Administrative Region

' Gerry E Johnson Optometrists, Brisbane, Australia

e Centre for Contact Lens Research, University of Waterloo, Waterloo, Ontario, Canada

${ }^{\mathrm{f}}$ Eye Hospital of Wenzhou Medical University, China

${ }^{\mathrm{g}}$ Contact Lens Section, Federal University of São Paulo/Paulista School of Medicine São Paulo, Brazil

${ }^{\mathrm{h}}$ Point Vision Bordeaux, Ophthalmologic Center, Bordeaux, France

${ }^{\mathrm{i}}$ Clinical and Experimental Optometry Research Lab (CEORLab) - Center of Physics, University of Minho, Portugal

j University of Alabama at Birmingham School of Optometry, Birmingham, AL, USA

${ }^{k}$ Menicon RED Innovation Centre, Fondation Pour Recherches Medicales, Geneva, Switzerland

${ }^{1}$ Clinica Schaefer, Curitiba, Parana, Brazil

${ }^{\mathrm{m}}$ International Association of Contact Lens Educators, Pune, India

${ }^{\mathrm{n}}$ Eye Research Institute Maastricht, University of Maastricht, Maastricht, the Netherlands

\section{A R T I C L E I N F O}

\section{Article history:}

Received 1 February 2016

Received in revised form 3 February 2016

Accepted 3 February 2016

\section{Keywords:}

Myopia control

Myopia progression

Myopia management

Orthokeratology

Global

Attitudes

\begin{abstract}
A B S T R A C T
Purpose: Myopia is a global public health issue; however, no information exists as to how potential myopia retardation strategies are being adopted globally.

Methods: A self-administrated, internet-based questionnaire was distributed in six languages, through professional bodies to eye care practitioners globally. The questions examined: awareness of increasing myopia prevalence, perceived efficacy and adoption of available strategies, and reasons for not adopting specific strategies.

Results: Of the 971 respondents, concern was higher (median 9/10) in Asia than in any other continent (7/ $10, \mathrm{p}<0.001)$ and they considered themselves more active in implementing myopia control strategies (8/ $10)$ than Australasia and Europe (7/10), with North (4/10) and South America (5/10) being least proactive $(\mathrm{p}<0.001)$. Orthokeratology was perceived to be the most effective method of myopia control, followed by increased time outdoors and pharmaceutical approaches, with under-correction and single vision spectacles felt to be the least effective $(\mathrm{p}<0.05)$. Although significant intra-regional differences existed, overall most practitioners $67.5( \pm 37.8) \%$ prescribed single vision spectacles or contact lenses as the primary mode of correction for myopic patients. The main justifications for their reluctance to prescribe alternatives to single vision refractive corrections were increased cost (35.6\%), inadequate information (33.3\%) and the unpredictability of outcomes (28.2\%).

Conclusions: Regardless of practitioners' awareness of the efficacy of myopia control techniques, the vast majority still prescribe single vision interventions to young myopes. In view of the increasing prevalence of myopia and existing evidence for interventions to slow myopia progression, clear guidelines for myopia management need to be established.
\end{abstract}

(c) 2016 British Contact Lens Association. Published by Elsevier Ltd. All rights reserved.

\footnotetext{
* Corresponding author at: Life and Health Sciences, Aston University, Aston

Triangle, Birmingham B4 7ET, UK.

E-mail address: j.s.w.wolffsohn@aston.ac.uk (J.S. Wolffsohn).
} 


\section{Introduction}

The prevalence of myopia has approximately doubled in the past three decades [1-3], arguably reaching epidemic levels. Prevalence rates of $70-87 \%$ have been reported amongst populations of schoolchildren and young adults in Asia [1,4-8], and around 20-50\% in America and Europe [9-12]. Moreover, the onset of myopia in the last two generations has been reported to occur earlier $[1,13,14]$ leading to an increased prevalence of high myopia $(\geq-6.00 \mathrm{D})$. High myopia is strongly associated with an increased risk of sight-threatening pathological ocular comorbidities, [1,15] including retinal detachment, glaucoma, and cataract [16-21]. A study conducted in Taiwan [1], comparing the age of onset and prevalence of myopia amongst schoolchildren from 1983 to 2000, shows an alarming shift towards a more myopic refractive error in recent years; in 1983, the mean onset of myopia was 11 years, whereas, in 2000, it was eight years; the mean refractive status observed at eight years of age was $0.45 \pm 1.03 \mathrm{D}$ and $-0.15 \pm 1.40 \mathrm{D}$ in 1983 and 2000, respectively, whereas at 11 years of age it was $-0.27 \pm 1.72 \mathrm{D}$ and $-1.20 \pm 1.93 \mathrm{D}$, respectively.

A range of factors including genetic predisposition [22-27], inadequate near accommodation response [28,29], elevated $\mathrm{AC} / \mathrm{A}$ ratio/esophoria [30,31], excessive time spent undertaking near work [25,32-34], low levels of outdoor activity [35-39], lighting levels $[36,40,41]$ and the magnitude of hyperopic peripheral defocus [42-50] have been linked to the development and/or progression of myopic refractive error. However, the exact mechanisms surrounding both myopia development and progression are not yet fully understood as the disease appears to be multifactorial in nature.

Over the past few years, there has been significant research and clinical interest in so-called 'myopia control' approaches, being clinical methods which are designed to be beneficial for attenuating childhood myopic progression. Sankaridurg and Holden [51] discussed the potential benefit that a six year-old east-Asian child with $-1.00 \mathrm{D}$ of myopia could have, at age 15 years, if myopia progression was reduced by $30.0 \%$, using an evidencebased model of progression rates of myopia. If this child's myopia progressed at the rate predicted by available natural history data, they would be expected to develop myopia in the order of $-7.00 \mathrm{D}$ by 15 years of age. If the myopia progression had been retarded by an estimated $30 \%$ over the eight-year follow-up period, then $-5.50 \mathrm{D}$ of myopia would be predicted. In a review paper, Flitcroft [17] highlighted that the higher the myopic refraction, the higher the odds ratio for myopic maculopathy, retinal detachment and, to a lesser extent, glaucoma and cataract. For example, compared to an emmetropes, the odds ratio for developing myopic maculopathy is 40.6 (95\% confidence interval: $13.3-124.4$ ) for myopia of -5.00 to $6.99 \mathrm{D}$, but increases to 126.8 (34.0-472.3) for myopia of -7.00 to $8.99 \mathrm{D}[17,21]$. Similarly, the odds ratio for developing retinal detachment is 21.5 (17.3-26.7) for myopia of -5.00 to $6.99 \mathrm{D}$, but increases to 44.2 (34.2-57.2) for myopia of -7.00 to $8.99 \mathrm{D}$ [17,52]. It has been estimated that reducing the rate of myopia progression by $33 \%$ would lead to a reduction of $73 \%$ in the frequency of high myopia (<-5.00 D) [53]. Lower levels of myopia have a reduced risk, but as the number of people with lower levels of myopia is greater, the public health risk of any myopia is still significant [17].

Multiple options are currently available for myopic refractive correction, including single vision, bifocal and progressive addition lens (PALs) spectacles, soft and rigid contact lenses (including orthokeratology) and refractive surgery. However, the relative contribution of these clinical methods for retarding myopia progression has only been more thoroughly investigated in more recent years (Fig. 1) [54-76].

It has been suggested that conventional single vision spectacle lenses may be ineffective for myopia control as they induce peripheral hyperopic defocus, a factor speculated to promote eye growth $[47,48,77,78]$. However some authors have questioned whether peripheral eye focus is the primary mechanism driving eye growth, as they reported that some myopic children wearing single vision spectacles had greater relative myopic defocus, and thus myopia progression was less than it was in those children wearing single vision spectacles with relatively greater hyperopic defocus [67,79]. Other large studies in humans have also found peripheral refraction to neither affect myopia onset or development $[64,80]$. Progressive addition and bifocal lenses have been

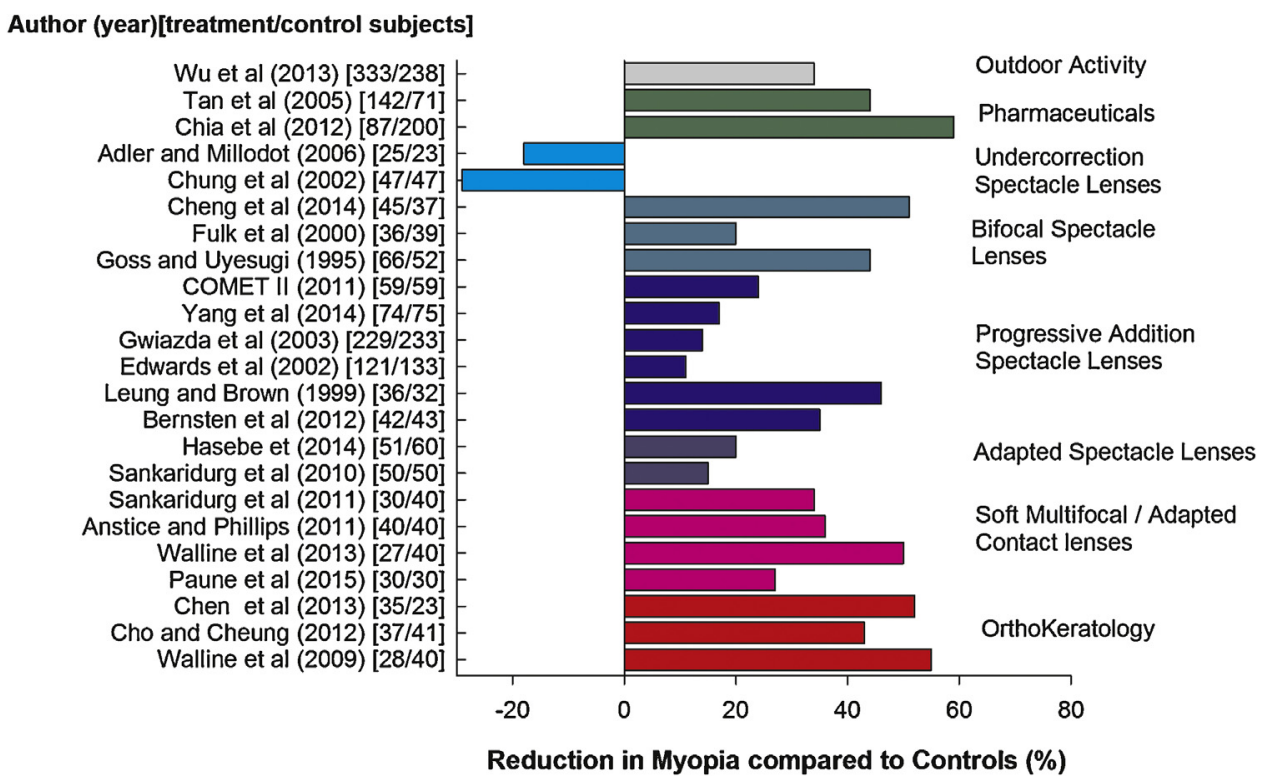

Fig. 1. Comparative studies [53-76] of the effectiveness (over the evaluated period) of different techniques to retard the progression of myopia. 
prescribed to reduce accommodative demand, and mitigate the blur associated with increased lag of accommodation in myopia $[28,29,31,64]$. It is presumed that an insufficient amount of accommodation might cause a relative retinal blur and, hence, be a triggering factor for axial elongation. The success rate of studies employing PALs and bifocals have varied from no effect $[65,81]$ to $46 \%$ for PALs (although this study was not randomised) [66], and 44-56\% for bifocals and executive bifocals [59,61]. However, other studies have reported retardation rates of $14 \%$ to $24 \%[60,64,66,82,83]$ which are less convincing.

Under-correction, which was believed not only to reduce accommodative demand, but induce myopic defocus, has been hypothesised to act as a halting signal to myopia development in animal studies [84,85]. Contrarily, under-correction has been found to accelerate the rate of myopia progression by $17-29 \%$ in human clinical studies [57,58].

Soft single vision contact lenses [86-88] and conventional rigid gas permeable (RGP) contact lenses $[87,89,90]$ have been found to have no effect on myopia progression. However, multifocal contact lens designs appear to be effective in reducing myopia progression (by $34-50 \%$ ) and may be more effective than bifocal or PAL spectacle lens designs; this may result from the optics of a contact lens, including the near portion, being consistently aligned with the position of gaze because the lens moves with the eye [64,71,91], and possibly more consistency in wearing time which seems to be an important factor for efficacy [92]. Orthokeratology studies are remarkably consistent in the level of myopia retardation shown, being around 50\% [74-76] and is considered at present the optical treatment with the strongest accumulated evidence [93].

Pharmaceutical treatment strategies, such as atropine $[56,81,94]$ and pirenzepine $[55,95,96]$, have shown high success rates ( $32 \%$ to $72 \%$ ). However, there has been a lack of consensus for the optimum concentration to prevent unwanted side-effects during treatment, and the rebound effect following cessation of treatment [55,59,83,97,98].

Epidemiology studies in the general population, and in monozygotic twins, have generally demonstrated that time spent outdoors reduces the likelihood of myopia onset [99-102]. The behavioural approach of increased outdoor activity has been shown to retard the onset of myopia by $11-34 \%$ [54]. It is postulated that the higher luminance levels, which exist outdoors compared to indoors, trigger the release of the retinal transmitter, dopamine, which is believed to prevent axial growth and myopia development $[36,40,41]$. In addition, it has been suggested that components of sunlight itself could activate particularly vitamin D, which could play a potential role in preventing eye growth $[36,103]$. Furthermore, viewing distances are generally greater outdoors compared to indoors, removing accommodative demand and retinal image quality could improve as pupil diameter is smaller in bright light, increasing depth of focus [38].

However, if and how these approaches are employed in clinical practice is still unclear. In 2013 the Vision Research Institute (Ferris State University Michigan Collage of Optometry) conducted a survey concerning the increasing rates of myopia prevalence (available online: http://www.myopiacontrol.org/ how-do-you-myopia-control-.html). Results showed that practitioners in United States of America were aware of the growing issue and tended to familiarise themselves with the current literature in the field. However, their rationale for prescribing, or the frequency of choosing different management strategies was not included in the survey. Likewise, Contact Lens Spectrum has also surveyed over 400 practitioners in the United States of America in both 2014 and 2015 showing that in both years, 24\% of practitioners report using contact lenses to control myopia; practitioners reported using soft multifocals and orthokeratology contact lens designs predominantly, with very few reporting rigid multifocals [104]. Several studies have reported statistically, but not clinically, significant reductions in the rates of myopia progression retardation (see Fig. 1) and some authors doubt the retardation effect achieved [60], presumably leaving practitioners confused and sceptical about the various management strategies available. Therefore, a better understanding of current trends of myopia management in clinical practice is required before targeted education and recommended criteria for intervention can be introduced.

\section{Method}

A self-administrated, internet-based cross-sectional survey in English, French, Spanish, Italian, Portuguese and Chinese was distributed using software SurveyMonkey (Palo Alto, California, USA) through various professional bodies across the world to reach eye care professionals (optometrists, dispensing opticians, ophthalmologists and others) globally. The survey comprised of nine questions relating to the self-reported clinical management behaviours of practitioners for progressive myopia and practitioner's current opinions on myopia related clinical care including:

- level of concern about the increasing frequency of paediatric myopia in their clinical practice (rated as 'Not at all,' to 'extremely,' on a 10 point scale)

- perceived effectiveness, defined as the expected level of reduction in childhood myopia progression of a range of myopia control options (rated as a percentage from 0 to 100\%)

- how active they would consider their clinical practice in the area of myopia control (rated as 'Not at all,' to 'fully,' on a 10 point scale)

- frequency of prescribing different myopia correction options for progressive/young myopes during a typical month

- minimum age a patient would need to be for them to consider myopia refractive correction options (assuming average handling skills and child/parent motivation)

- minimum amount of myopia that would need to be present to consider myopia refractive correction options (specified in half dioptre steps)

- minimum level of myopia progression that would prompt a practitioner to specifically adopt a myopia control approach (specified in quarter dioptre steps)

- frequency of adopting single vision under-correction as a strategy to slow myopia progression (reported as 'no,' 'sometimes,' or 'always')?

- if they had only ever fitted single vision spectacles/contact lenses for myopic patients, what had prevented them (multiple options could be selected) from prescribing alternative refractive correction methods; options consisted of:

- They don't believe that these are any more effective

- The outcome is not predictable

- Safety concerns

- Cost to the patient makes them uneconomical

- Additional chair time required

- Inadequate information/knowledge

- Benefit/risk ratio

- Other

There was an option to add further comments to each of the questions and the topic as a whole. Voluntary participation in the survey, following an explanation of the research, was anonymous, however, respondents were asked to provide basic demographic information about themselves (highest qualification, years of being qualified and everyday working environment). The data was collected between January and June 2015. 


\subsection{Statistical analysis}

Statistical analysis was conducted with SPSS (v21 IBM, New York, USA). Only complete surveys were analysed. Median, mean and standard deviations were calculated for each question response, with the results grouped by continent (Asia, Australasia, Europe, North America and South America) and countries within a continent where response rate allowed $(n \geq 30)$, with KruskalWallis tests applied to determine statistical difference (taken as $\mathrm{p}<0.05$ ) between them. For conciseness, only significant comparisons have been reported.

\section{Results}

\subsection{Responses}

The total number of 971 complete survey responses were received, with the distribution by continent being: Africa 7 (not included in further analysis), Asia 291, Australasia 119; Europe 339; North America 133; and South America 82. Country specific responses could be extracted from:

- Europe: France $(n=34)$, Italy $(n=72)$, Netherlands $(n=38)$ Portugal $(n=48)$,

- Spain $(n=34)$ and UK/EIRE $(n=52)$

- Asia: China ( $n=137)$, Hong Kong $(n=61)$ and India $(n=37)$

- North America; Canada $(n=33)$ and USA $(n=100)$

Of the study participants, $72.4 \%(n=698)$ were optometrists, $18.6 \%(n=180)$ were ophthalmologists, $5.8 \%(n=56)$ were contact lens opticians and $3.2 \%(n=31)$ were other types of eye care specialists. The principal working environment for $84.4 \%$ was in clinical practice $(n=814), 11.3 \%$ worked in academia $(n=109), 1.6 \%$ worked within industry $(n=16)$ and $2.7 \%(n=26)$ worked in other environments. However, all study participants were registered eye care practitioners. The median number of years qualified was the 11-20 category, with a normally distributed spread.

\subsection{Self-reported concern about the increasing frequency of paediatric myopia (Fig. 2)}

Practitioners' concern about increasing frequencies of paediatric myopia in their practices was higher (median 9/10) in Asia than any of the other continents $(p<0.001)$, with a similar level of concern (all with a median of $7 / 10 ; p>0.05$ ) across Australasia, Europe, North and South America. In Asia, Chinese practitioners were more concerned $(8.8 \pm 1.5)$ than those in Hong Kong $(7.9 \pm 1.7 ; \mathrm{p}=0.001)$ or India $(7.3 \pm 2.6 ; \mathrm{p}=0.002)$. In Europe,

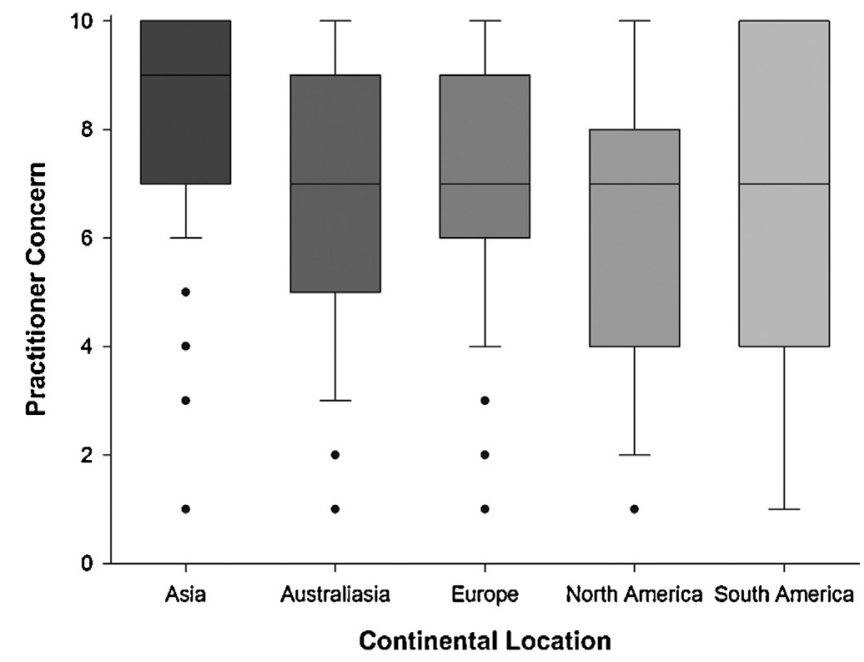

Fig. 2. Level of practitioner concern (rated from 0 to 10) regarding the perceived increasing frequency of paediatric myopia in their practice for practitioners located in different continents. $\mathrm{N}=964$. Error bars $=1 \mathrm{SD}$.

Portuguese $(8.2 \pm 3.2)$ and Spanish $(8.3 \pm 2.3)$ practitioners were more concerned than those in Italy $(6.9 \pm 2.5 ; \mathrm{p}=0.046$, $\mathrm{p}=0.027$ respectively), the Netherlands $(6.3 \pm 2.1 ; \mathrm{p}=0.002$, $\mathrm{p}=0.001)$ or the UK/EIRE $(5.8 \pm 2.6 ; \mathrm{p}<0.001, \mathrm{p}<0.001)$. In North America, practitioners from the USA $(6.8 \pm 2.7)$ were more concerned than their Canadian neighbours $(5.4 \pm 2.7 ; \mathrm{p}=0.005)$.

\subsection{Perceived effectiveness of myopia control options (Table 1)}

Overall, orthokeratology was perceived by practitioners to be the most effective method of myopia control, followed by increased time outdoors and pharmaceutical approaches. Single vision distance under-correction and single vision spectacles were perceived to be the least effective method. These findings were consistent across all continents except for South America $(\mathrm{p}<0.05)$, where all the modalities were perceived to be similarly effective (12-24\%), except for time spent outdoors (35\%). Compared with practitioners from all other continents, Asians practitioners considered single vision, bifocal and progression addition lenses to be relatively more effective for reducing childhood myopia progression $(\mathrm{p}<0.01)$. Australian and North American practitioners perceived single vision contact lenses as less effective than practitioners from other continents $(\mathrm{p}<0.01)$. North American practitioners had less confidence in orthokeratology and pharmaceuticals as appropriate methods for myopia control than those from Asia or Australasia ( $p=0.001)$; the same

Table 1

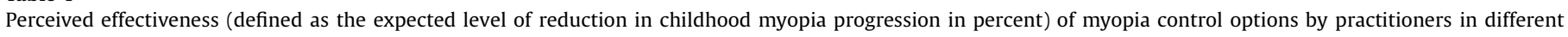
continents. Data are expressed as mean \pm S.D.

\begin{tabular}{|c|c|c|c|c|c|c|}
\hline \multicolumn{7}{|l|}{ Continent } \\
\hline \multicolumn{2}{|l|}{ Technique } & \multirow{2}{*}{$\begin{array}{l}\text { Asia } \\
6.5 \pm 13.9\end{array}$} & \multirow{2}{*}{$\frac{\text { Australasia }}{2.5 \pm 7.4}$} & \multirow{2}{*}{$\begin{array}{l}\text { Europe } \\
6.4 \pm 15.8\end{array}$} & \multirow{2}{*}{$\frac{\text { North America }}{2.9 \pm 7.9}$} & \multirow{2}{*}{$\frac{\text { South America }}{13.4 \pm 23.1}$} \\
\hline Spectacles & Under-correction & & & & & \\
\hline & Single Vision & $16.0 \pm 23.6$ & $4.2 \pm 12.5$ & $10.0 \pm 21.8$ & $4.0 \pm 14.0$ & $18.1 \pm 30.7$ \\
\hline & Bifocals & $18.4 \pm 21.1$ & $14.1 \pm 14.8$ & $12.4 \pm 17.5$ & $11.6 \pm 14.4$ & $12.3 \pm 24.2$ \\
\hline & Progressive Addition (PALs) & $21.3 \pm 21.2$ & $16.0 \pm 14.0$ & $14.7 \pm 18.6$ & $11.3 \pm 13.5$ & $12.8 \pm 24.8$ \\
\hline \multirow[t]{5}{*}{ Contact Lenses } & Rigid Gas Permeable (RGP) & $23.9 \pm 26.9$ & $9.6 \pm 13.8$ & $14.1 \pm 20.8$ & $9.9 \pm 15.4$ & $13.6 \pm 27.0$ \\
\hline & Single Vision Soft & $11.9 \pm 20.6$ & $4.1 \pm 11.5$ & $10.1 \pm 20.5$ & $2.9 \pm 10.5$ & $16.0 \pm 29.0$ \\
\hline & Multifocal Soft & $15.5 \pm 20.2$ & $22.5 \pm 19.3$ & $16.4 \pm 25.7$ & $18.4 \pm 20.5$ & $11.5 \pm 19.7$ \\
\hline & Novel Myopia Control Soft & $24.4 \pm 26.0$ & $29.1 \pm 19.3$ & $25.2 \pm 25.7$ & $21.5 \pm 23.1$ & $18.8 \pm 28.5$ \\
\hline & Orthokeratology & $48.6 \pm 29.6$ & $47.8 \pm 25.3$ & $44.3 \pm 29.0$ & $36.9 \pm 30.1$ & $23.9 \pm 32.3$ \\
\hline & $31.7 \pm 27.8$ & $39.0 \pm 32.4$ & $24.2 \pm 29.4$ & $21.8 \pm 27.0$ & $14.6 \pm 23.3$ \\
\hline \multicolumn{2}{|c|}{ Refractive Surgery } & $17.4 \pm 29.7$ & $11.4 \pm 24.3$ & $12.8 \pm 25.6$ & $13.5 \pm 30.6$ & $18.0 \pm 29.4$ \\
\hline \multicolumn{2}{|c|}{ Increased Time Outdoors } & $38.7 \pm 27.5$ & $29.7 \pm 22.0$ & $29.4 \pm 26.2$ & $20.5 \pm 17.9$ & $35.3 \pm 32.0$ \\
\hline
\end{tabular}




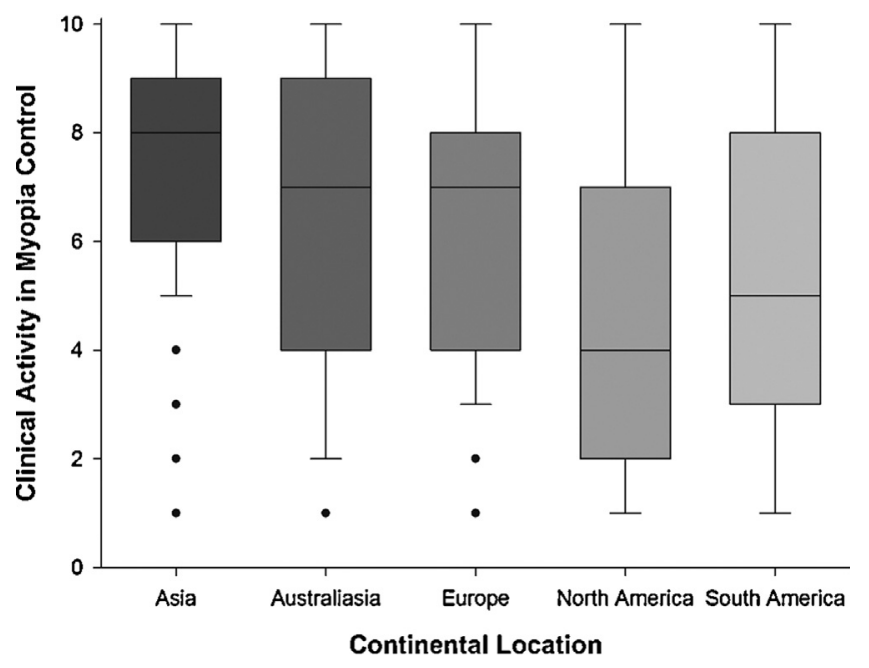

Fig. 3. Perceived level of clinical activity in the area of myopia control for practitioners located in different continents. $\mathrm{N}=964$. Error bars $=1 \mathrm{SD}$.

was true of European practitioners with respect to pharmaceuticals $(\mathrm{p}<0.001)$. North Americans were also more sceptical about the potential benefit of increasing time spent outdoors on myopia progression compared with practitioners from other continents $(\mathrm{p}<0.05)$. Intra-region comparisons showed that there were wide variations in perceived effectiveness across all myopia control options .

\subsection{Perceived level of clinical activity in the area of myopia control} (Fig. 3)

Asian practitioners considered their clinical practice of myopia control to be more active (median 8/10) than practitioners from Australasian (median $7 / 10 ; \mathrm{p}=0.028$ ), European (median $7 / 10$; $\mathrm{p}<0.001$ ), North American (median $4 / 10$; $\mathrm{p}<0.001$ ) or South American practitioners (median 5/10, $\mathrm{p}<0.001$ ). North American practitioners perceived themselves to be relatively less active in this area of practice than those from Europe $(p<0.001)$ and Australasia ( $p<0.001)$. Within Europe there were no differences between countries, however, within Asia, Indian practitioners $(6.05 \pm 1.99)$ considered themselves relatively less active than Chinese $(7.96 \pm 1.96 ; \mathrm{p}<0.001)$ or Hong Kong $(7.31 \pm 2.20$; $\mathrm{p}=0.002$ ) practitioners. Within North America, Canadian practitioners $(3.97 \pm 3.42)$ considered themselves less active than those from the USA $(4.96 \pm 2.83 ; \mathrm{p}=0.034)$.

\subsection{Frequency of prescribing different myopia correction options for progressing/young myopes (Table 2)}

The majority of progressing myopes were being prescribed either single vision (full correction) spectacles $(47.8 \pm 31.7 \%)$ or single vision contact lenses $(15.2 \pm 17.3 \%)$. Orthokeratology $(14.3 \pm 24.3 \%)$, PAL spectacles $(6.5 \pm 14.3 \%)$, RGPs $(4.5 \pm 10.5 \%)$, multifocal contact lenses $(4.1 \pm 11.3 \%)$, bifocal spectacles $(2.6 \pm 8.2 \%)$, novel myopia control contact lenses $(2.1 \pm 7.9 \%)$, pharmaceuticals $(1.9 \pm 8.7 \%)$ and refractive surgery $(1.0 \pm 5.4 \%)$ were utilised relatively less frequency. Asian practitioners indicated prescribing single vision (full correction) spectacles most frequently, whereas those from Australia prescribed them least often ( $p<0.001$ ). North American practitioners indicated prescribing bifocal spectacles most frequently $(p<0.001$ ) for progressing/ young myopes. Australian practitioners, and to a lesser degree, Asian practitioners, prescribed PALs more frequently $(\mathrm{p}<0.001)$ than those from other continents. Australians and North American practitioners prescribed RGPs $(\mathrm{p}<0.001)$ less frequently to these patients. Asian practitioners prescribe single vision contact lenses $(\mathrm{p}<0.001)$ least often, while North American practitioners prescribed more standard multifocal contact lenses $(\mathrm{p}<0.001)$ than other regions. Few novel myopia control soft contact lenses are being prescribed in any continent. South American practitioners prescribed orthokeratology least frequently $(\mathrm{p}<0.001)$. Asian practitioners indicated utilising pharmaceuticals more frequently $(\mathrm{p}<0.001)$ than practitioners from other continents for progressing/young myopes. Asian and South American practitioners recommended refractive surgery more than other continents for these patients, but the prescribing frequency was still low ( $\mathrm{p}<0.001)$. Intra-region comparison showed large variations in prescribing habits for all myopia control options .

\subsection{Minimum patient age that practitioners consider myopia correction options (Table 3)}

Single vision spectacles were prescribed from the youngest age ( $5.4 \pm 1.5$ years). Multifocal spectacles (bifocal: $6.3 \pm 2.3$ years; PAL: $7.3 \pm 2.8$ years) and pharmaceuticals ( $6.4 \pm 2.6$ years) tended to be reserved for older children. Contact lenses were only considered appropriate for older children, especially those intended specifically for myopia control (single vision: $6.5 \pm 3.4$ years; novel myopia control soft: $8.8 \pm 3.1$ years; orthokeratology: $8.8 \pm 3.1$ years; multifocal: $8.9 \pm 3.1$ years; RGPs $9.9 \pm 3.3$ years). Most practitioners did not recommend refractive surgery to patients under 18 years of age. For single vision spectacles, bifocal spectacles and PALs, European and Asian practitioners were more conservative in their minimum fitting age $(\mathrm{p}<0.05)$ than Australasian or North American practitioners. Asian and South American

Table 2

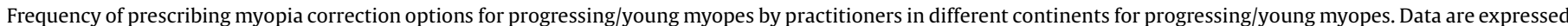
as mean \pm S.D.

\begin{tabular}{|c|c|c|c|c|c|c|}
\hline \multicolumn{7}{|l|}{ Continent } \\
\hline Technique & & Asia & Australasia & Europe & North America & South America \\
\hline \multirow[t]{3}{*}{ Spectacles } & Single Vision & $57.6 \pm 31.3$ & $36.8 \pm 30.2$ & $42.2 \pm 30.7$ & $49.6 \pm 31.3$ & $52.1 \pm 30.5$ \\
\hline & Bifocals & $2.9 \pm 7.3$ & $1.3 \pm 4.4$ & $2.1 \pm 7.0$ & $5.1 \pm 13.6$ & $1.9 \pm 7.0$ \\
\hline & Progressive Addition (PALs) & $7.4 \pm 13.3$ & $17.4 \pm 23.0$ & $4.1 \pm 12.2$ & $3.7 \pm 9.2$ & $1.8 \pm 5.2$ \\
\hline \multirow[t]{5}{*}{ Contact Lenses } & Rigid Gas Permeable (RGP) & $4.9 \pm 8.5$ & $0.6 \pm 2.1$ & $6.1 \pm 13.6$ & $2.4 \pm 8.3$ & $6.8 \pm 10.8$ \\
\hline & Single Vision Soft & $5.7 \pm 9.9$ & $13.9 \pm 13.4$ & $20.2 \pm 18.8$ & $18.8 \pm 16.5$ & $21.0 \pm 20.3$ \\
\hline & Multifocal Soft & $0.8 \pm 2.9$ & $6.2 \pm 11.9$ & $4.3 \pm 11.0$ & $8.5 \pm 17.5$ & $2.1 \pm 7.2$ \\
\hline & Novel Myopia Control Soft & $2.2 \pm 8.1$ & $1.5 \pm 4.7$ & $2.4 \pm 8.8$ & $0.9 \pm 5.1$ & $3.0 \pm 10.6$ \\
\hline & Orthokeratology & $11.1 \pm 17.6$ & $21.2 \pm 29.1$ & $18.3 \pm 27.6$ & $9.4 \pm 18.5$ & $7.9 \pm 25.2$ \\
\hline Pharmaceutical & & $5.6 \pm 14.5$ & $0.8 \pm 3.3$ & $0.1 \pm 1.2$ & $1.1 \pm 6.8$ & $0.7 \pm 5.0$ \\
\hline Refractive Surgery & & $2.0 \pm 8.3$ & $0.3 \pm 1.3$ & $0.3 \pm 1.6$ & $0.6 \pm 4.5$ & $2.8 \pm 7.6$ \\
\hline
\end{tabular}


Table 3

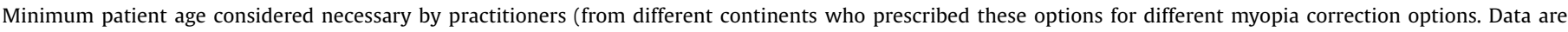
expressed as mean \pm S.D years (\% that would not prescribe this refractive modality).

\begin{tabular}{|c|c|c|c|c|c|c|}
\hline \multicolumn{7}{|l|}{ Continent } \\
\hline Technique & & Asia & Australasia & Europe & North America & South America \\
\hline \multirow[t]{3}{*}{ Spectacles } & Single Vision & $5.9 \pm 3.9(1)$ & $5.3 \pm 0.5(6)$ & $7.4 \pm 3.0(6)$ & $5.2 \pm 0.6(4)$ & $5.5 \pm 1.2(11)$ \\
\hline & Bifocals & $6.6 \pm 2.6(37)$ & $6.0 \pm 1.3(32)$ & $7.4 \pm 2.6(48)$ & $5.1 \pm 0.5(21)$ & $7.5 \pm 2.9(66)$ \\
\hline & Progressive Addition (PALs) & $7.8 \pm 3.0(21)$ & $6.5 \pm 1.4(14)$ & $7.8 \pm 2.8(47)$ & $6.7 \pm 2.9(23)$ & $8.0 \pm 3.2(65)$ \\
\hline \multirow[t]{5}{*}{ Contact Lenses } & Rigid Gas Permeable (RGP) & $10.1 \pm 3.3(20)$ & $9.0 \pm 1.7(32)$ & $7.9 \pm 2.4(22)$ & $9.3 \pm 3.0(24)$ & $10.2 \pm 4.3(24)$ \\
\hline & Single Vision Soft & $10.9 \pm 3.8(24)$ & $8.3 \pm 0.8(8)$ & $7.8 \pm 2.7(8)$ & $7.9 \pm 2.4(7)$ & $10.3 \pm 3.7(11)$ \\
\hline & Multifocal Soft & $11.1 \pm 4.0(52)$ & $8.3 \pm 0.8(22)$ & $7.4 \pm 2.5(45)$ & $8.1 \pm 2.7(24)$ & $11.0 \pm 3.6(63)$ \\
\hline & Specific Myopia Control Soft & $10.8 \pm 3.5(30)$ & $8.3 \pm 0.8(25)$ & $7.3 \pm 2.5(32)$ & $7.9 \pm 2.6(33)$ & $10.3 \pm 3.9(45)$ \\
\hline & Orthokeratology & $9.6 \pm 3.2(8)$ & $8.0 \pm 1.1(13)$ & $8.1 \pm 2.3(17)$ & $8.0 \pm 3.1(30)$ & $12.3 \pm 4.8(49)$ \\
\hline Pharmaceutical & & $6.4 \pm 2.6(44)$ & $6.7 \pm 3.9(65)$ & $7.9 \pm 3.6(87)$ & $6.4 \pm 3.3(62)$ & $6.3 \pm 2.2(64)$ \\
\hline Refractive Surgery & & $16.9 \pm 2.9(50)$ & $18.0 \pm 0.0(83)$ & $12.8 \pm 4.5(83)$ & $18.0 \pm 0.0(71)$ & $15.5 \pm 5.2(56)$ \\
\hline
\end{tabular}

practitioners were more conservative in their minimum fitting age of all soft contact lens modalities than Australian, European or North American practitioners $(\mathrm{p}<0.01)$. There was no difference between regions in the minimum age they would consider prescribing RGP contact lenses, pharmaceuticals or refractive surgery correction options ( $p>0.05)$.

Within Europe, practitioners from the Netherlands were the most conservative in their minimum age for fitting single vision spectacles $(\mathrm{p}<0.001)$. French practitioners were most conservative in their minimum age for fitting single vision contact lenses compared to those from the rest of the continent, with the exception of Portuguese practitioners, who were conservative with their minimum fitting age of all types of contact lenses $(p<0.05)$. Indian practitioners were more conservative than Chinese practitioners on the minimum fitting age for RGPs ( $p=0.003)$, whereas Chinese practitioners were more conservative in their minimum fitting age for single vision soft contact lenses than those from either Hong Kong or India $(p<0.05)$. Orthokeratology was considered at an earlier age in Hong Kong than India or China $(\mathrm{p}<0.001)$ and in the USA than in Canada $(\mathrm{p}=0.029)$. Pharmaceuticals were considered at an earlier age in China than in Hong Kong or India $(\mathrm{p}=0.001)$.

\subsection{Minimum degree of myopia that needs to be present for practitioners to consider myopia control options (Table 4)}

Overall practitioners indicated that myopia would be corrected with single vision spectacles at a lower degree $(-1.07 \pm 0.90 \mathrm{D})$ than it would with refractive surgery $(-3.06 \pm 1.62 \mathrm{D})$. All other modalities would be considered at approximately $-2.00 \mathrm{D}$. Asian practitioners required a higher level of myopic refractive error before they would consider single vision spectacles than all other regions $(p<0.01)$. Australasian practitioners were willing to fit bifocals and PALs at a lower level of myopia than Asian, European or South American clinicians $(\mathrm{p}<0.01)$. North American practitioners prescribed bifocal and PALs to children with a lower degree of myopia than those from Asia $(p=0.001)$. However, Asian practitioners would consider single vision soft contact lenses, novel myopia control contact lenses, orthokeratology and pharmaceutical intervention at a lower level of myopia than those from Australasia or Europe $(p<0.01)$, and single vision soft contact lenses and pharmaceutical treatment at a lower level of myopia than those from North and South America $(p<0.01)$. Multifocal contact lenses were considered at a lower level of myopia by Australasian practitioners than those from Asia, Europe or South America $(p<0.01)$. Asian practitioners required a higher level of refractive error before they would consider fitting RGPs than those from Europe and North America $(\mathrm{p}<0.01)$. Asian and European practitioners would consider recommending refractive surgery at a significantly higher level of myopia than those from Australasia, North or South America $(\mathrm{p}<0.05)$. Within Europe, Portuguese and French practitioners required a higher level of refractive error before they would consider fitting RGPs than those from other European nations $(p<0.05)$. Portuguese practitioners also required a higher level of refractive error before they would consider orthokeratology $(\mathrm{p}<0.01)$ or refractive surgery $(\mathrm{p}<0.001)$ than practitioners from other European nations. Indian practitioners required a higher level of refractive error before they would consider bifocals $(p<0.05)$, PALs $(p<0.01)$ or orthokeratology $(p=0.001)$ than practitioners from China or Hong Kong. Chinese practitioners considered prescribing pharmaceuticals to children with a lower level of myopia $(-0.66 \pm 0.4 \mathrm{D})$ compared to practitioners from India $(-2.86 \pm 1.01 \mathrm{D})$ or Hong Kong $(-2.39 \pm 1.75 \mathrm{D} ; \mathrm{p}<0.001)$. The only difference across North

Table 4

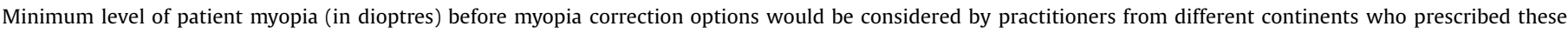
options. Data are expressed as mean \pm S.D.

\begin{tabular}{|c|c|c|c|c|c|c|}
\hline \multicolumn{7}{|l|}{ Continent } \\
\hline Technique & & Asia & Australasia & Europe & North America & South America \\
\hline \multirow[t]{3}{*}{ Spectacles } & Single Vision & $-1.2 \pm 1.0$ & $-0.8 \pm 0.3$ & $-0.8 \pm 0.9$ & $-0.8 \pm 0.7$ & $-1.3 \pm 0.4$ \\
\hline & Bifocals & $-1.8 \pm 1.1$ & $-0.8 \pm 0.4$ & $-1.8 \pm 1.4$ & $-1.1 \pm 0.7$ & $1.5 \pm 0.0$ \\
\hline & Progressive Addition (PALs) & $-2.1 \pm 1.4$ & $-0.9 \pm 0.6$ & $-1.8 \pm 1.4$ & $-1.1 \pm 0.7$ & $-1.5 \pm 0.0$ \\
\hline \multirow[t]{5}{*}{ Contact Lenses } & Rigid Gas Permeable (RGP) & $-3.1 \pm 1.9$ & $-2.8 \pm 1.4$ & $-2.2 \pm 1.8$ & $-1.5 \pm 1.4$ & $-1.8 \pm 0.4$ \\
\hline & Single Vision Soft & $-2.6 \pm 1.8$ & $-1.8 \pm 0.9$ & $-1.1 \pm 0.8$ & $-0.9 \pm 0.7$ & $-1.5 \pm 0.0$ \\
\hline & Multifocal Soft & $-2.6 \pm 1.7$ & $-1.8 \pm 1.0$ & $-1.9 \pm 1.5$ & $-1.1 \pm 0.7$ & $-1.5 \pm 0.0$ \\
\hline & Novel Myopia Control Soft & $-2.7 \pm 1.8$ & $-1.7 \pm 0.9$ & $-1.9 \pm 1.5$ & $-1.5 \pm 1.2$ & $-1.5 \pm 0.0$ \\
\hline & Orthokeratology & $-2.4 \pm 1.5$ & $-1.6 \pm 0.8$ & $-2.3 \pm 1.6$ & $-1.5 \pm 1.2$ & $-1.5 \pm 0.0$ \\
\hline Pharmaceutical & & $-1.6 \pm 1.5$ & $-2.1 \pm 0.9$ & $-3.1 \pm 1.6$ & $-1.5 \pm 1.3$ & $-2.5 \pm 2.1$ \\
\hline Refractive Surgery & & $-3.5 \pm 1.6$ & $-2.9 \pm 1.5$ & $-2.9 \pm 1.5$ & $-1.7 \pm 1.3$ & $-2.8 \pm 1.8$ \\
\hline
\end{tabular}


America was that Canadian practitioners required a higher level of refractive error before they would consider PALs $(p=0.012)$ than those from the USA.

3.8. Minimum annual amount of patient myopia progression that would prompt a practitioner to specifically adopt a myopia control approach (Fig. 4)

The minimum myopia progression rate that practitioners considered warranted a myopia control approach was $0.51-0.75 \mathrm{D} /$ year for the majority of respondents (31.1\%), with $74 \%$ indicating a level between 0.25 and 1.00 D/year. Australian practitioners indicated that they would adopt myopia control strategies for myopia progressing at slower rates than practitioners from Asia, North or South American $(\mathrm{p}<0.001)$. European practitioners would be willing to treat myopia progression at slower rates than those from Asian $(\mathrm{p}<0.001)$ or South American $(p=0.003)$. There were no differences in the minimum annual myopia progression triggering a myopia control approach between Europe $(p=0.090)$, Asia $(p=0.365)$ or North America $(p=0.057)$. Other factors influencing practitioners' management decisions, as identified from the free text responses, included family history of myopia (6 respondents), age of myopia onset (10 respondents), absolute degree of refractive error at the time ( 2 respondents), ocular biometry (3 respondents), environmental factors/lifestyle (6 respondents), lighting exposure (3 respondents) and parental decisions (3 respondents).

\subsection{Use of single-vision under-correction as a strategy to slow myopia progression (Fig. 5)}

Overall, most practitioners did not consider single-vision distance under-correction to be an effective strategy for attenuating myopia progression (72.7\%). South American practitioners used this strategy relatively more than those from Australia, Asia or North America $(\mathrm{p}<0.01)$. Within Europe, Spanish and Portuguese practitioners indicated using under-correction as a strategy to control myopia more than those from the UK and EIRE $(\mathrm{p}<0.05)$. Within Asia, Indian practitioners utilised under-correction more than those from China or Hong Kong $(\mathrm{p}<0.001)$. Within North America, there was no difference in the use of under-correction between the USA and Canada $(\mathrm{p}=0.719)$.

\subsection{Factors preventing the prescription of a myopia control approach (Fig. 6)}

The most common reasons practitioners gave for not adopting myopia control strategies were: they were felt to be uneconomical (35.6\%); they considered there to be inadequate information about the modalities (33.3\%); they viewed the outcomes to be unpredictable (28.2\%); concerns about safety (25.3\%); they perceived them to be ineffective for reducing myopia progression (23.8\%); and the benefit to risk ratio was too low (20.5\%). There was no significant difference in the distribution of these factors between or within continents $(p>0.05)$. Free text comments identified other factors affecting the prescription of these strategies to relate to the relative availability of the myopia control treatments and the instrumentation necessary to prescribe them, and the need for consistent regulations and informational materials .

\section{Discussion}

This is the first study to examine the self-reported attitudes and practices of eye care practitioners towards myopia control approaches across the globe. Close to one thousand practitioners responded, principally spread over five continents. The exact response rate is not known, as maximum coverage was promoted by involving professional bodies whose members may not all be practicing eye care practitioners. However, it may be presumed that questionnaires are completed both by people cynical and enthusiastic to the issue being examined, balancing the average response. In addition, the recruitment approach across nations was the same, allowing cross-national comparisons. The majority of the

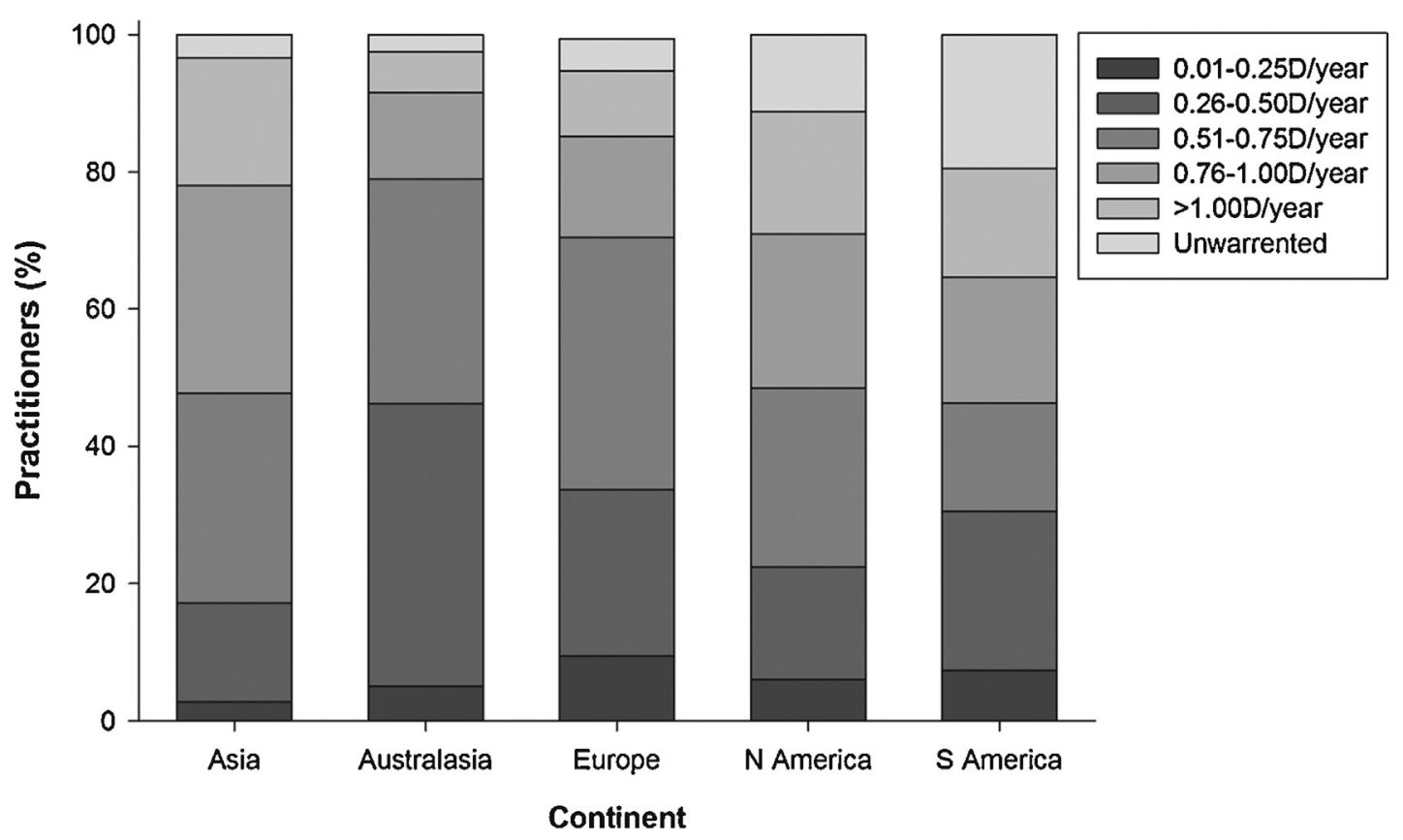

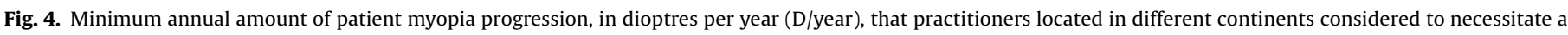
myopia control approach. $\mathrm{N}=964$. 


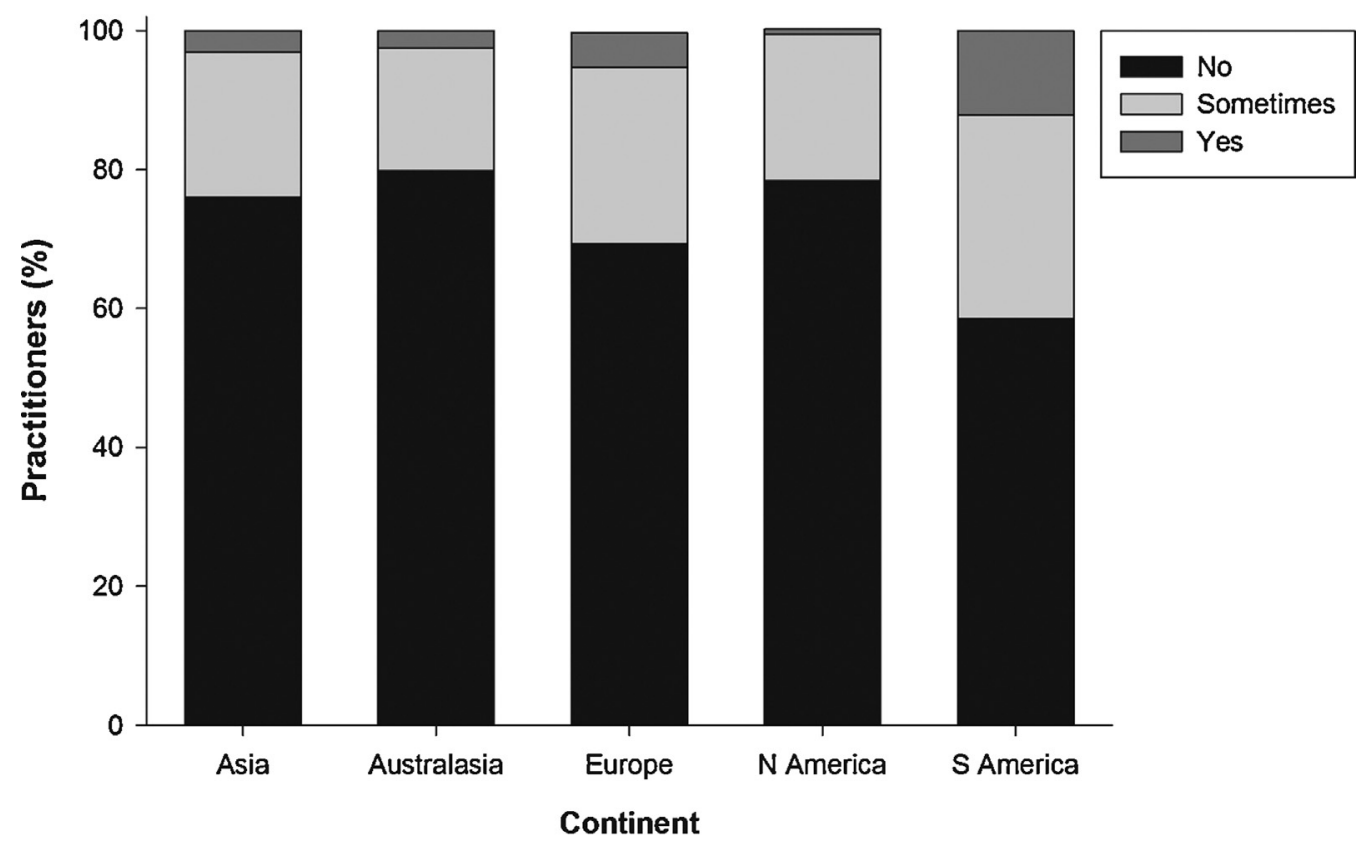

Fig. 5. Use of single-vision distance under-correction as a strategy to slow myopia progression by practitioners located in different continents. $\mathrm{N}=964$.

respondents (91.0\%) were optometrists and ophthalmologists, reflecting those professions legally allowed to prescribe vision care correction and, in many regions, pharmaceuticals as well.

As one might expect from the high prevalence rates of myopia in Asia, Asian practitioners, especially those practicing in China, were more concerned about the increasing prevalence of paediatric myopia in their practices than clinicians in any of the other continents. A similar pattern existed in relation to how active they considered their clinical practice in the area of myopia control. Myopia prevalence was approximately $30 \%$ in $30-35$ year olds in Spain [105] and may be increasing in Portugal [106], but is as high as $58 \%$ in Italian university students and $28 \%$ in Dutch school children [107]; hence it is unclear why the former country's practitioners are more concerned than the latter. The prevalence of myopia in the USA was around 30\% [11], but the myopia occurrence is not documented in Canada to warrant their lower concern.

Orthokeratology was correctly perceived by eye care practitioners to be one of the most effective methods for attenuating childhood myopia progression. However, the effectiveness of pharmaceutical approaches were underestimated by practitioners and increased time spent outdoors overestimated compared to current evidence (Fig. 1). Differences in lifestyle, such as population density might affect perceived effectiveness of approached such as perceived time spent outdoors. While singlevision distance under-correction has been shown fairly conclusively to increase, rather than decrease, the rate of myopia

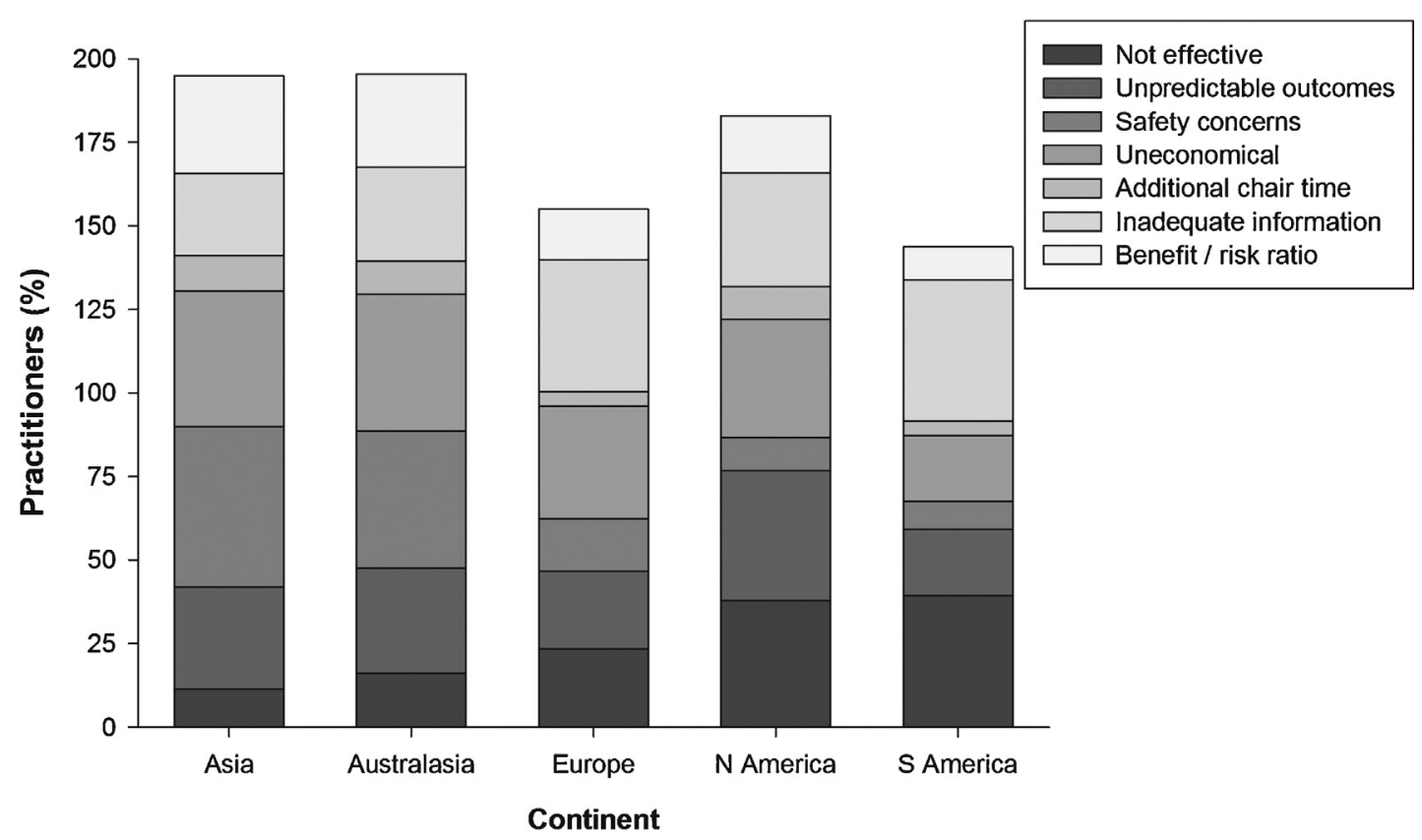

Fig. 6. Factors preventing practitioners located in different continents from prescribing a myopia control approach. $\mathrm{N}=964$. 
progression in children [57,58], there were still practitioners who consider the converse to be true; this was confirmed by a question later in the survey, with under-correction still practiced as a method of myopia control particularly by practitioners from South America, Spain and Portugal within Europe and India within Asia.

Despite the self-perceived activity of practitioners in the area of myopia control, over two thirds of progressing and/or young myopes were being prescribed single vision spectacles or contact lenses (68\%), with continental and national differences in the adoption of refractive correction options known to reduce myopia progression. Approximately one third of practitioners not adopting myopia control approaches felt them to be uneconomical and/or that there was inadequate information about them; about one quarter of respondents suggested that outcomes were unpredictable and/or that myopia control methods were ineffective, with some also being concerned about the relative safety of these strategies. Further comments raised the issue of availability of some myopia control options, presumably of novel myopia control lenses, as current approaches are all off-label, highlighting the need for regulatory oversight and guidance. Limited access to necessary instrumentation was also raised as a potential barrier, as more advanced contact lens fittings, such as orthokeratology, require the use of corneal topography. Attempts to specifically manipulate peripheral retinal focus may also require instrumentation to rapidly and robustly assess peripheral retinal shape and/ or refraction with myopia control ophthalmic medical devices. However, this strategy might not 'translate' well from animal studies to human trials $[79,80]$.

Spherical equivalent refractive error (measured under cycloplegia) is currently the single best predictive measure of juvenile myopia development, with children aged six years with less than $+0.75 \mathrm{D}$ of hyperopia being at increased risk of developing myopia [108]. Most practitioners were comfortable fitting refractive corrections with basic optical designs and even pharmaceuticals to myopic patients of this age, but tended to wait until a child was older for more complex designs such as PALs, novel myopia control soft contact lenses and RGPs (including orthokeratology). Interestingly, one potential advantage of orthokeratology is that the parents or carer can manage lens application, removal and lens care, along with the lenses not having to leave the home, which can make this modality a popular option for parents or carers with younger myopic children. This is exemplified by Hong Kong, an early adopter of orthokeratology, where its use is considered at an earlier age than other countries in the region.

Research suggests that lower levels of hypermetropia at a young age is a strong risk factor for myopia development, so it would seem that practitioners are far too conservative in waiting until mild-moderate levels of myopia are present before control approaches are considered $[80,108]$. Myopia progresses at much faster rates in children in comparison to teenagers, thus supporting the need for earlier intervention [109]. There may also "window of opportunity" for myopia treatment according to the age of onset, rate of progression and myopia magnitude [110]. More research is needed on the relative benefits of myopia control strategies in adolescents and even young adults. Practitioners located in Asia considered most myopia control approaches at a lower level of myopia than other continents, which is presumably due to this continent having one of the highest prevalences of myopia worldwide. Interestingly, Chinese practitioners considered prescribing pharmaceutical modalities at a younger age, and at a much lower level of myopia, compared with practitioners from other countries in the region. This may be due to different countries having different regulations and practitioners with different background (for example training, education and scope of practice), which can affect local practice, apart from the prevalence of myopia and need for correction or retardation. The rate of patient refractive progression that triggered practitioners to prescribe a myopia control approach largely mirrored the prevalence rate of myopia in each region; the higher the prevalence of myopia, the higher the level of myopia developed in individuals and the higher the risk of ocular pathology [20]. Practitioners understandably also identified several other factors that, combined with the degree of myopic progression, influenced their decision to prescribe myopia control approaches; these included family history, age of onset, absolute amount of refractive error at that time, ocular biometry, environmental factors/lifestyle, lighting exposure and the degree of parental support [111].

In conclusion, this global survey of current trends in eye care practitioner myopia management attitudes and strategies in clinical practice has identified that, despite growing evidence of the negative impact of even low levels of myopia on health economics, and moderate levels of practitioner concern and perceived activity (particularly where the prevalence of myopia is highest), uptake of appropriate techniques is generally poor. Furthermore, myopia control techniques are not being applied early enough in a child's ocular development to elicit their optimum effect. Adequate education of practitioners is lacking, along with access to appropriately regulated myopia control 'labelled' products with efficacy and safety data. A guide needs to be developed to inform practitioners of economically viable models of eye care, including the development of instrumentation to enhance management selection, which address the myopia epidemic to reduce the growing health burden.

\section{Acknowledgement}

This project was supported by many eye care organisations across the globe and in particular the British Contact Lens Association.

This project was not funded externally and none of the authors or organisations involved have any financial conflicts.

\section{References}

[1] L.L. Lin, Y.F. Shih, C.K. Hsiao, C.J. Chen, Prevalence of myopia in Taiwanese schoolchildren: 1983 to 2000, Ann. Acad. Med. Singapore 33 (2004) 27-33.

[2] C.W. Pan, D. Ramamurthy, S.M. Saw, Worldwide prevalence and risk factors for myopia, Ophthalmic Physiol. Opt. 32 (2012) 3-16.

[3] S. Vitale, R.D. Sperduto, F.L. Ferris, Increased prevalence of myopia in the United States between 1971-1972 and 1999-2004, Arch. Ophthalmol. 127 (2009) 1632-1639.

[4] M.H. Edwards, C.S. Lam, The epidemiology of myopia in Hong Kong, Ann. Acad. Med. Singapore 33 (2004) 34-38.

[5] W.S. Goh, C.S. Lam, Changes in refractive trends and optical components of Hong Kong Chinese aged 19-39 years, Ophthalmic Physiol. Opt. 14 (1994) 378-382.

[6] M. He, J. Zeng, Y. Liu, J. Xu, G.P. Pokharel, L.B. Ellwein, Refractive error and visual impairment in urban children in southern China, Invest. Ophthalmol. Vis. Sci. 45 (2004) 793-799.

[7] L.L. Lin, C.J. Chen, P.T. Hung, L.S. Ko, Nation-wide survey of myopia among schoolchildren in Taiwan, 1986, Acta Ophthalmol. Suppl. (Oxf) 185 (1988) 29 33.

[8] P.W. Ting, C.S. Lam, M.H. Edwards, K.L. Schmid, Prevalence of myopia in a group of Hong Kong microscopists, Optom. Vis. Sci. 81 (2004) 88-93.

[9] N.S. Logan, P. Shah, A.R. Rudnicka, B. Gilmartin, C.G. Owen, Childhood ethnic differences in ametropia and ocular biometry: the Aston Eye Study, Ophthalmic Physiol. Opt. 31 (2011) 550-558.

[10] R.D. Sperduto, D. Seigel, J. Roberts, M. Rowland, Prevalence of myopia in the United States, Arch. Opththalmol. 101 (1983) 405-407.

[11] S. Vitale, L. Ellwein, M.F. Cotch, F.L. Ferris, R. Sperduto, Prevalence of refractive error in the United States, 1999-2004, Arch. Opththalmol. 126 (2008) 1111 1119.

[12] K.M. Williams, V.J.M. Verhoeven, P. Cumberland, G. Bertelsen, C. Wolfram, G. H.S. Buitendijk, A. Hofman, C.M. Van Duijn, J.R. Vingerling, R. Kuijpers, R. Hohn, A. Mirshahi, A.P. Khawaja, R.N. Luben, M.G. Erke, T. Von Hanno, O Mahroo, R. Hogg, C. Gieger, A. Cougnard-Gregoire, E. Anastasopoulos, A. Bron, J.F. Dartigues, J.F. Korobelnik, C. Creuzot-Garcher, F. Topouzis, C. Delcourt, J. Rahi, T. Meitinger, A. Fletcher, P.J. Foster, N. Pfeiffer, C.C.W. Klaver, C.J. 
Hammond, Prevalence of refractive error in Europe: the European Eye Epidemiology (E-3) Consortium, Eur. J. Epidemiol. 30 (2015) 305-315.

[13] Y.B. Liang, Z. Lin, B. Vasudevan, V. Jhanji, A. Young, T.Y. Gao, S.S. Rong, N.L. Wang, K.J. Ciuffreda, Generational difference of refractive error in the baseline study of the Beijing Myopia Progression Study, Br. J. Ophthalmol. 97 (2013) 765-769.

[14] H.J. Lin, L. Wan, F.J. Tsai, Y.Y. Tsai, L.A. Chen, A.L. Tsai, Y.C. Huang, Overnight orthokeratology is comparable with atropine in controlling myopia, BMC Ophthalmol. 14 (2014).

[15] C.S.Y. Lam, C.H. Lam, S.C.K. Cheng, L.Y.L. Chan, Prevalence of myopia among Hong Kong Chinese schoolchildren: changes over two decades, Ophthalmic Physiol. Opt. 32 (2012) 17-24.

[16] N. Brown, A.R. Hill, Cataract: the relation between myopia and cataract morphology, Br. J. Ophthalmol. 71 (1987) 405-414.

[17] D.I. Flitcroft, The complex interactions of retinal, optical and environmental factors in myopia aetiology, Prog. Retin. Eye Res. 31 (2012) 622-660.

[18] M.C. Leske, A. Connell, S.Y. Wu, L.G. Hyman, A.P. Schachat, Risk factors for open-angle glaucoma: the Barbados Eye Study, Arch. Ophthalmol. 113 (1995) 918-924.

[19] P. Mitchell, F. Hourihan, J. Sandbach, J.J. Wang, The relationship between glaucoma and myopia: the Blue Mountains Eye Study, Ophthalmology 106 (1999) 2010-2015.

[20] S.M. Saw, G. Gazzard, E.C. Shih-Yen, W.H. Chua, Myopia and associated pathological complications, Ophthalmic Physiol. Opt. 25 (2005) 381-391.

[21] J. Vongphanit, P. Mitchell, J.J. Wang, Prevalence and progression of myopic retinopathy in an older population, Ophthalmology 109 (2002) 704-711.

[22] C.Y. Cheng, M. Schache, M.K. Ikram, T.L. Young, J.A. Guggenheim, V. Vitart, S. Macgregor, V.J. Verhoeven, V.A. Barathi, J. Liao, P.G. Hysi, J.E. Bailey-Wilson, B. St. Pourcain, J.P. Kemp, G. Mcmahon, N.J. Timpson, D.M. Evans, G.W. Montgomery, A. Mishra, Y.X. Wang, J.J. Wang, E. Rochtchina, O. Polasek, A.F Wright, N. Amin, E.M. Van Leeuwen, J.F. Wilson, C.E. Pennell, C.M. Van Duijn, P.T. De Jong, J.R. Vingerling, X. Zhou, P. Chen, R. Li, W.T. Tay, Y. Zheng, M. Chew, E. Consortium for Refractive, Myopia, K.P. Burdon, J.E. Craig, S.K. Iyengar, R.P Igo, Jr., J.H. Lass, Jr., G. Fuchs' Genetics Multi-Center Study, E.Y. Chew, T. Haller E. Mihailov, A. Metspalu, J. Wedenoja, C.L. Simpson, R. Wojciechowski, R. Hohn, A. Mirshahi, T. Zeller, N. Pfeiffer, K.J. Lackner, C. Wellcome Trust Case Control, T. Bettecken, T. Meitinger, K. Oexle, M. Pirastu, L. Portas, A. Nag, K.M. Williams, E. Yonova-Doing, R Klein, B.E. Klein, S.M. Hosseini, A.D. Paterson, C Diabetes, I. Complications Trial/Epidemiology of Diabetes, G. Complications Research, K.M. Makela, T. Lehtimaki, M. Kahonen, O. Raitakari, N. Yoshimura, F. Matsuda, L.J. Chen, C.P. Pang, S.P. Yip, M.K. Yap, A. Meguro, N. Mizuki, H. Inoko, P.J. Foster, J.H. Zhao, E. Vithana, E.S. Tai, Q. Fan, L., Xu, H. Campbell, B. Fleck, I. Rudan, T. Aung, A. Hofman, A.G. Uitterlinden, G.Bencic, C.C. Khor, H., Forward, O. Parssinen, P. Mitchell, F. Rivadeneira, A.W. Hewitt, C. Williams, B. A. Oostra, Y.Y. Teo, C.J. Hammond, D. Stambolian, D.A. Mackey, C.C. Klaver, T.Y Wong, S.M. Saw, P.N. Baird, Nine loci for ocular axial length identified through genome-wide association studies, including shared loci with refractive error Am. J. Hum. Genet., 93 (2013) 264-277.

[23] J.E. Farbrother, G. Kirov, M.J. Owen, R. Pong-Wong, C.S. Haley, J.A Guggenheim, Linkage analysis of the genetic loci for high myopia on 18p 12q and $17 q$ in 51 UK families, Invest. Ophthalmol. Vis. Sci. 45 (2004) 2879-2885.

[24] D. Kurtz, L. Hyman, J.E. Gwiazda, R. Manny, L.M. Dong, Y. Wang, M. Scheiman, Role of parental myopia in the progression of myopia and its interaction with treatment in COMET children, Invest. Ophthalmol. Vis. Sci. 48 (2007) 562.

[25] D.O. Mutti, G.L. Mitchell, M.L. Moeschberger, L.A. Jones, K. Zadnik, Parental myopia near work, school achievement, and children's refractive error, Invest. Ophthalmol. Vis. Sci. 43 (2002) 3633-3640.

[26] R. Pacella, J. Mclellan, K. Grice, E.A. Del Bono, J.L. Wiggs, J.E. Gwiazda, Role of genetic factors in the etiology of juvenile-onset myopia based on a longitudinal study of refractive error, Optom. Vis. Sci. 76 (1999) 381-386.

[27] K. Zadnik, Myopia development in childhood, Optom. Vis. Sci. 74 (1997) 603608.

[28] J. Gwiazda, F. Thorn, J. Bauer, R. Held, Myopic children show insufficient accommodative response to blur, Invest. Ophthalmol. Vis. Sci. 34 (1993) 690694.

[29] J. Gwiazda, J. Bauer, F. Thorn, R. Held, A dynamic relationship between myopia and blur-driven accommodation in school-aged children, Vision Res. 35 (1995) 1299-1304

[30] B. Drobe, R. Desaintandre, The pre-myopic syndrome, Ophthalmic Physiol. Opt. 15 (1995) 375-378.

[31] J. Gwiazda, K. Grice, F. Thorn, Response AC/A ratios are elevated in myopic children, Ophthalmic Physiol. Opt. 19 (1999) 173-179.

[32] G.C. Ashton, Nearwork, school achievement and myopia, J. Biosoc. Sci. 17 (1985) 223-233.

[33] J. Gwiazda, L. Deng, L. Dias, W. Marsh-Tootle, C.S. Grp, Association of education and occupation with myopia in COMET parents, Optom. Vis. Sci. 88 (2011) 1045-1053.

[34] J.M. Ip, S.M. Saw, K.A. Rose, I.G. Morgan, A. Kijley, J.J. Wang, P. Mitchell, Role of near work in myopia: findings in a sample of Australian school children, Invest. Ophthalmol. Vis. Sci. 49 (2008) 2903.

[35] M. Dirani, L. Tong, G. Gazzard, X. Zhang, A. Chia, T.L. Young, K.A. Rose, P. Mitchell, S.-M. Saw, Outdoor activity and myopia in Singapore teenage children, Br. J. Ophthalmol. 93 (2009) 997-1000.

[36] A.N. French, R.S. Ashby, I.G. Morgan, K.A. Rose, Time outdoors and the prevention of myopia, Exp. Eye Res. 114 (2013) 58-68.
[37] J.A. Guggenheim, K. Northstone, G. Mcmahon, A.R. Ness, K. Deere, C. Mattocks, B. St Pourcain, C. Williams, Time outdoors and physical activity as predictors of incident myopia in childhood: a prospective cohort study, Invest. Ophthalmol. Vis. Sci. 53 (2012) 2856.

[38] K.A. Rose, I.G. Morgan, J. Ip, A. Kifley, S. Huynh, W. Smith, P. Mitchell, Outdoor activity reduces the prevalence of myopia in children, Ophthalmology 115 (2008) 1279-1285.

[39] K.A. Rose, I.G. Morgan, W. Smith, G. Burlutsky, P. Mitchell, S.M. Saw, Myopia lifestyle, and schooling in students of Chinese ethnicity in Singapore and Sydney, Arch. Ophthalmol. 126 (2008) 527-530.

[40] R. Ashby, A. Ohlendorf, F. Schaeffel, The effect of ambient illuminance on the development of deprivation myopia in chicks, Invest. Ophthalmol. Vis. Sci. 50 (2009) 5348-5354.

[41] R.S. Ashby, F. Schaeffel, The effect of bright light on lens compensation in chicks, Invest. Ophthalmol. Vis. Sci. 51 (2010) 5247-5253.

[42] J. Hoogerheide, F. Rempt, W.P.H. Hoogenboom, Acquired myopia in young pilots, Ophthalmologica 163 (1971) 209-215.

[43] N.S. Logan, B. Gilmartin, M. Dunne, Computation of retinal contour in anisomyopia, Ophthalmic Physiol. Opt. 15 (1995) 363-366.

[44] M. Millodot, Effect of ametropia on peripheral refraction, Am. J. Optom. Physiol. Opt. 58 (1981) 691-695.

[45] D.O. Mutti, J.R. Hayes, G.L. Mitchell, L.A. Jones, M.L. Moeschberger, S.A. Cotter, R.N. Kleinstein, R.E. Manny, J.D. Twelker, K. Zadnik, Refractive error, axial length, and relative peripheral refractive error before and after the onset of myopia, Invest. Ophthalmol. Vis. Sci. 48 (2007) 2510.

[46] C. Mutti, L.A. Jones, M.L. Moeschberger, K. Zadnik, AC/A ratio, age, and refractive error in children, Am. J. Ophthalmol. 130 (2000) 690.

[47] E.L. Smith, Prentice award lecture 2010: a case for peripheral optical treatment strategies for myopia, Optom. Vis. Sci. 88 (2011) 1029-1044.

[48] E.L. Smith, L.F. Hung, J. Huang, Relative peripheral hyperopic defocus alters central refractive development in infant monkeys, Vision Res. 49 (2009) 2386-2392.

[49] E.L. Smith, C.S. Kee, R. Ramamirtham, Y. Qiao-Grider, L.F. Hung, Peripheral vision can influence eye growth and refractive development in infant monkeys, Invest. Ophthalmol. Vis. Sci. 46 (2005) 3965-3972.

[50] E.L. Smith, R. Ramamirtham, Y. Qiao-Grider, L.F. Hung, J. Huang, C.S. Kee, D. Coats, E. Paysse, Effects of foveal ablation on emmetropization and formdeprivation myopia, Invest. Ophthalmol. Vis. Sci. 48 (2007) 3914-3922.

[51] P. Sankaridurg, B. Holden, Practical applications to modify and control the development of ametropia, Eye 28 (2014) 134-141.

[52] A. Ogawa, M. Tanaka, The relationship between refractive errors and retinal detachment-analysis of 1,166 retinal detachment cases, Jpn. J. Ophthalmol. 32 (1987) 310-315.

[53] N.A. Brennan, Predicted reduction in high myopia for various degrees of myopia control, Cont. Lens Anterior Eye 35 (2012) e14-e15.

[54] P.C. Wu, C.L. Tsai, H.L. Wu, Y.H. Yang, H.K. Kuo, Outdoor activity during class recess reduces myopia onset and progression in school children, Ophthalmology 120 (2013) 1080-1085.

[55] D.T. Tan, D.S. Lam, W.H. Chua, D.F. Shu-Ping, R.S. Crockett, A.P.S. Group, Oneyear multicenter double-masked, placebo-controlled, parallel safety and efficacy study of $2 \%$ pirenzepine ophthalmic gel in children with myopia, Ophthalmology 112 (2005) 84-91.

[56] A. Chia, W.H. Chua, Y.B. Cheung, W.L. Wong, A. Lingham, A. Fong, D. Tan, Atropine for the treatment of childhood myopia: safety and efficacy of $0.5 \%$, $0.1 \%$, and $0.01 \%$ doses (Atropine for the Treatment of Myopia 2), Ophthalmology 119 (2012) 347-354.

[57] D. Adler, M. Millodot, The possible effect of undercorrection on myopic progression in children, Clin. Exp. Optom. 89 (2006) 315-321.

[58] K. Chung, N. Mohidin, D.J. O'leary, Undercorrection of myopia enhances rather than inhibits myopia progression, Vision Res. 42 (2002) 2555-2559.

[59] D. Cheng, G.C. Woo, B. Drobe, K.L. Schmid, Effect of bifocal and prismatic bifocal spectacles on myopia progression in children three-year results of a randomized clinical trial, JAMA Ophthalmol. 132 (2014) 258-264.

[60] G.W. Fulk, L.A. Cyert, D.E. Parker, A randomized trial of the effect of singlevision vs. bifocal lenses on myopia progression in children with esophoria, Optom. Vis. Sci. 77 (2000) 395-401.

[61] D.A. Goss, E. Uyesugi, Effectiveness of bifocal control of childhood myopia progression as a function of near point phoria and binocular cross-cylinder, J. Optom. Vis. Dev. 26 (1995) 12-17.

[62] J. Gwiazda, D.L. Chandler, S.A. Cotter, D.F. Everett, L. Hyman, B.M. Kaminski, M.T. Kulp, D.W. Lyon, R.E. Manny, W.L. Marsh-Tootle, N.S. Matta, B.M. Melia, T. T. Norton, M.M. Scheiman, D.I. Silbert, A.E.M. Weissberg, Progressiveaddition lenses versus single-vision lenses for slowing progression of myopia in children with high accommodative lag and near esophoria, Invest. Ophthalmol. Vis. Sci. 52 (2011) 2749-2757.

[63] Z.K. Yang, W.H. Lan, J. Ge, W. Liu, X. Chen, L.X. Chen, M.B. Yu, The effectiveness of progressive addition lenses on the progression of myopia in Chinese children, Ophthalmic Physiol. Opt. 29 (2009) 41-48.

[64] J. Gwiazda, L. Hyman, M. Hussein, D. Everett, T.T. Norton, D. Kurtz, M.C. Leske, R. Manny, W. Marsh-Tootle, M. Scheiman, A randomized clinical trial of progressive addition lenses versus single vision lenses on the progression of myopia in children, Invest. Ophthalmol. Vis. Sci. 44 (2003) 1492-1500.

[65] M.H. Edwards, R.W.H. Li, C.S.Y. Lam, J.K.F. Lew, B.S.Y. Yu, The Hong Kong progressive lens myopia control study: study design and main findings, Invest. Ophthalmol. Vis. Sci. 43 (2002) 2852-2858. 
[66] J.T.M. Leung, B. Brown, Progression of myopia in Hong Kong Chinese schoolchildren is slowed by wearing progressive lenses, Optom. Vis. Sci. 76 (1999) 346-354.

[67] D.A. Berntsen, L.T. Sinnott, D.O. Mutti, K. Zadnik, A randomized trial using progressive addition lenses to evaluate theories of myopia progression in children with a high lag of accommodation, Invest. Ophthalmol. Vis. Sci. 53 (2012) 640.

[68] S. Hasebe, J. Jun, S.R. Varnas, Myopia control with positively aspherized progressive addition lenses: a 2-year multicenter, randomized, controlled trial, Invest. Ophthalmol. Vis. Sci. 55 (2014) 7177-7188.

[69] P. Sankaridurg, L. Donovan, S. Varnas, A. Ho, X. Chen, A. Martinez, S. Fisher, Z Lin, E.L. Smith 3rd, J. Ge, B. Holden, Spectacle lenses designed to reduce progression of myopia: 12-month results, Optom. Vis. Sci. 87 (2010) 631-641.

[70] P. Sankaridurg, B. Holden, E. Smith, T. Naduvilath 3rd, X. Chen, P.L. De La Jara, A. Martinez, J. Kwan, A. Ho, K. Frick, J. Ge, Decrease in rate of myopia progression with a contact lens designed to reduce relative peripheral hyperopia: one-year results, Invest. Ophthalmol. Vis. Sci. 52 (2011) 9362 9367.

[71] N.S. Anstice, J.R. Phillips, Effect of dual-focus soft contact lens wear on axial myopia progression in children, Ophthalmology 118 (2011) 1152-1161.

[72] J.J. Walline, K.L. Greiner, M.E. Mcvey, L.A. Jones-Jordan, Multifocal contact lens myopia control, Optom. Vis. Sci. 90 (2013) 1207-1214.

[73] J. Pauné, H. Morales, J. Armengol, L. Quevedo, M. Faria-Ribeiro, J.M. GonzálezMéijome, Myopia control with a novel peripheral gradient soft lens and orthokeratology: a 2-year clinical trial, Biomed. Res. Int. 2015 (2015).

[74] C. Chen, S.W. Cheung, P. Cho, Myopia control using toric orthokeratology (TOSEE study), Invest. Ophthalmol. Vis. Sci. 54 (2013) 6510-6517.

[75] P. Cho, S.W. Cheung, Retardation of myopia in Orthokeratology (ROMIO) study: a 2-year randomized clinical trial, Invest. Ophthalmol. Vis. Sci. 53 (2012) 7077-7085.

[76] J.J. Walline, L.A. Jones, L.T. Sinnott, Corneal reshaping and myopia progression, Br. J. Ophthalmol. 93 (2009) 1181-1185.

[77] Z. Lin, A. Martinez, X. Chen, L. Li, P. Sankaridurg, B.A. Holden, J. Ge, Peripheral defocus with single-vision spectacle lenses in myopic children, Optom. Vis. Sci. 87 (2010) 4-9.

[78] E.L. Smith, J. Huang, L.F. Hung, T.L. Blasdel, T.L. Humbird, K.H. Bockhorst, Hemiretinal form deprivation: evidence for local control of eye growth and refractive development in infant monkeys, Invest. Ophthalmol. Vis. Sci. 50 (2009) 5057-5069.

[79] D.A. Atchison, S.-M. Li, H. Li, S.-Y. Li, L.-R. Liu, M.-T. Kang, B. Meng, Y.-Y. Sun, S.Y. Zhan, P. Mitchell, Relative peripheral hyperopia does not predict development and progression of myopia in children, Invest. Ophthalmol. Vis. Sci. 56 (2015) 6162-6170.

[80] D.O. Mutti, L.T. Sinnott, G.L. Mitchell, L.A. Jones-Jordan, M.L. Moeschberger, S. A. Cotter, R.N. Kleinstein, R.E. Manny, J.D. Twelker, K. Zadnik, Relative peripheral refractive error and the risk of onset and progression of myopia in children, Invest. Ophthalmol. Vis. Sci. 52 (2011) 199.

[81] Y.F. Shih, C.K. Hsiao, C.J. Chen, C.W. Chang, P.T. Hung, L.L.K. Lin, An intervention trial on efficacy of atropine and multi-focal glasses in controlling myopic progression, Acta Ophthalmol. Scand. 79 (2001) 233-236.

[82] S. Hasebe, H. Ohtsuki, T. Nonaka, C. Nakatsuka, M. Miyata, I. Hamasaki, S. Kimura, Effect of progressive addition lenses on myopia progression in Japanese children: a prospective randomized, double-masked, crossover trial, Invest. Ophthalmol. Vis. Sci. 49 (2008) 2781-2789.

[83] Z. Yang, W. Lan, J. Ge, W. Liu, X. Chen, L. Chen, M. Yu, The effectiveness of progressive addition lenses on the progression of myopia in Chinese children, Ophthalmic Physiol. Opt. 29 (2009) 41-48.

[84] A.W. Shaikh, J.T. Siegwart Jr., T.T. Norton, Effect of interrupted lens wear on compensation for a minus lens in tree shrews, Optom. Vis. Sci. 76 (1999) 308 315.

[85] E.L. Smith, L.F. Hung, R.S. Harwerth, Developmental visual system anomalies and the limits of emmetropization, Ophthalmic Physiol. Opt. 19 (1999) 90102.

[86] D.G. Horner, P.S. Soni, T.O. Salmon, T.S. Swartz, Myopia progression in adolescent wearers of soft contact lenses and spectacles, Optom. Vis. Sci. 76 (1999) 474-479.

[87] J.J Walline, L.A. Jones, D.O. Mutti, K. Zadnik, A randomized trial of the effects of rigid contact lenses on myopia progression, Arch. Ophthalmol. 122 (2004) $1760-1766$.

[88] J.J. Walline, L.A. Jones, L. Sinnott, R.E. Manny, A. Gaume, M.J. Rah, M. Chitkara, S. Lyons, A randomized trial of the effect of soft contact lenses on myopia progression in children, Invest. Ophthalmol. Vis. Sci. 49 (2008) 4702-4706.
[89] J. Katz, O.D. Schein, B. Levy, T. Cruiscullo, S.-M. Saw, U. Rajan, T.-K. Chan, C.Y. Khoo, S.-J. Chew, A randomized trial of rigid gas permeable contact lenses to reduce progression of children's myopia, Am. J. Ophthalmol. 136 (2003) 82-90.

[90] C.Y. Khoo, J. Chong, U. Rajan, A 3-year study on the effect of RGP contact lenses on myopic children, Singapore Med. J. 40 (1999) 230-237.

[91] T.A. Aller, C. Wildsoet, Bifocal soft contact lenses as a possible myopia control treatment: a case report involving identical twins, Clin. Exp. Optom. 91 (2008) 394-399.

[92] C.S.Y. Lam, W.C. Tang, D.Y.Y. Tse, Y.Y. Tang, C.H. To, Defocus Incorporated Soft Contact (DISC) lens slows myopia progression in Hong Kong Chinese schoolchildren: a 2-year randomised clinical trial, Br. J. Ophthalmol. 98 (2014) 40-45.

[93] J.M. Gonzalez-Meijome, G. Carracedo, D. Lopes-Ferreira, M.A. Faria-Ribeiro, S C. Peixoto-De-Matos, A. Queiros, Stabilization in early adult-onset myopia with corneal refractive therapy, Cont. Lens Anterior Eye 39 (2016) 72-77.

[94] W.-H. Chua, V. Balakrishnan, Y.-H. Chan, L. Tong, Y. Ling, B.-L. Quah, D. Tan, Atropine for the treatment of childhood myopia, Ophthalmology 113 (2006) 2285-2291.

[95] R.M. Siatkowski, S. Cotter, J.M. Miller, C.A. Scher, R.S. Crockett, G.D. Novack, Safety and efficacy of $2 \%$ pirenzepine ophthalmic gel in children WithMyopia: a 1-year multicenter, double-masked, placebo-controlled parallel study, Arch. Ophthalmol. 122 (2004) 1667-1674.

[96] R.M. Siatkowski, S.A. Cotter, R. Crockett, J.M. Miller, G.D. Novack, K. Zadnik, U. P.S. Group, Two-year multicenter randomized, double-masked, placebocontrolled, parallel safety and efficacy study of $2 \%$ pirenzepineophthalmicgelinchildrenwithmyopia, J. AAPOS 12 (2008) 332339.

[97] A. Chia, Q.S. Lu, D. Tan, Five-year clinical trial on atropine for the treatment of myopia 2: myopia control with atropine $0.01 \%$ eyedrops, Ophthalmology (2015).

[98] L. Tong, X.L. Huang, A.L. Koh, X. Zhang, D.T. Tan, W.-H. Chua, Atropine for the treatment of childhood myopia: effect on myopia progression after cessation of atropine, Ophthalmology 116 (2009) 572-579.

[99] Y.Y. Lee, C.T. Lo, S.J. Sheu, J.L. Lin, What factors are associated with myopia in young adults? A survey study in Taiwan Military Conscripts, Invest. Ophthalmol. Vis. Sci. 54 (2013) 1026-1033.

[100] O. Parssinen, M. Kauppinen, A. Viljanen, The progression of myopia from its onset at age 8-12 to adulthood and the influence of heredity and external factors on myopic progression. A 23-year follow-up study, Acta Ophthalmol 92 (2014) 730-739.

[101] R. Ramessur, K.M. Williams, C.J. Hammond, Risk factors for myopia in a discordant monozygotic twin study, Ophthalmic Physiol. Opt. 35 (2015) 643651.

[102] J.C. Sherwin, M.H. Reacher, R.H. Keogh, A.P. Khawaja, D.A. Mackey, P.J. Foster, The association between time spent outdoors and myopia in children and adolescents a systematic review and meta-analysis, Ophthalmology 119 (2012) 2141-2151.

[103] D.O. Mutti, M.E. Cooper, E. Dragan, L.A. Jones-Jordan, M.D. Bailey, M.L. Marazita, J.C. Murray, K. Zadnik, C.S. Grp, Vitamin d receptor (VDR) and group-specific component (GC, vitamin D-binding protein) polymorphisms in myopia, Invest. Ophthalmol. Vis. Sci. 52 (2011) 3818-3824.

[104] J.J. Nichols, Contact lenses 2015, Contact Lens Spectrum 31 (2016) 18-23.

[105] R. Montes-Mico, T. Ferrer-Blasco, Distribution of refractive errors in Spain, Doc. Ophthalmol. 101 (2000) 25-33.

[106] J. Jorge, J.B. Almeida, M.A. Parafita, Refractive, biometric and topographic changes among Portuguese university science students: a 3-year longitudinal study, Ophthalmic Physiol. Opt. 27 (2007) 287-294.

[107] T.J.W. Hendricks, J. De Brabander, M.H.P. Vankan-Hendricks, F.G. Van Der Horst, F. Hendrikse, J.A. Knottnerus, Prevalence of habitual refractive errors and anisometropia among Dutch schoolchildren and hospital employees, Acta Ophthalmol. 87 (2009) 538-543.

[108] K. Zadnik, L.T. Sinnott, S.A. Cotter, L.A. Jones-Jordan, R.N. Kleinstein, R.E. Manny, J.D. Twelker, D.O. Mutti, E. Collaborative Longitudinal, Prediction of juvenile-onset myopia, JAMA Ophthalmol. 133 (2015) 683-689.

[109] L.M. Dong, M. Fazzari, J. Gwiazda, L. Hyman, T. Norton, F. Thorn, Q.H. Zhang, C. S. Grp, Myopia stabilization and associated factors among participants in the correction of myopia evaluation trial (COMET), Invest. Ophthalmol. Vis. Sci. 54 (2013) 7871-7883.

[110] F. Thorn, J. Gwiazda, R. Held, Myopia progression is specified by a double exponential growth function, Optom. Vis. Sci. 82 (2005) 286-297.

[111] S.W. Cheung, C. Lam, P. Cho, Parents' knowledge and perspective of optical methods for myopia control in children, Optom. Vis. Sci. 91 (2014) 634-641. 